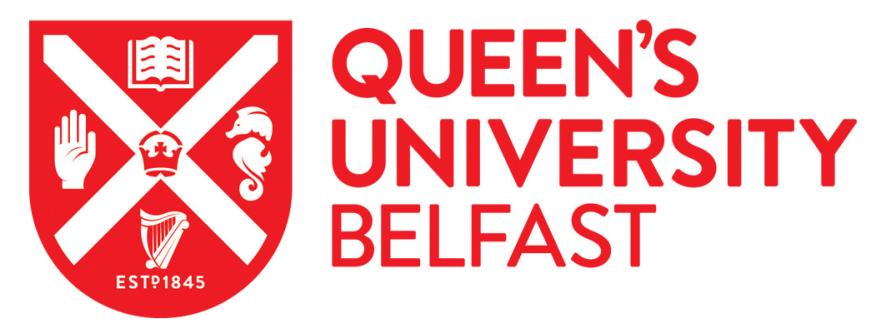

\title{
On the statistics of transverse permeability of randomly distributed fibers
}

Bodaghi, M., Catalanotti, G., \& Correia, N. (2016). On the statistics of transverse permeability of randomly distributed fibers. Composite Structures, 158, 323-332. https://doi.org/10.1016/j.compstruct.2016.09.045

\author{
Published in: \\ Composite Structures
}

\section{Document Version:}

Peer reviewed version

Queen's University Belfast - Research Portal:

Link to publication record in Queen's University Belfast Research Portal

\section{Publisher rights}

(C) 2016 Elsevier Ltd. This manuscript version is made available under the CC-BY-NC-ND 4.0 license http://creativecommons.org/licenses/bync-nd/4.0/,which permits distribution and reproduction for non-commercial purposes, provided the author and source are cited.

\section{General rights}

Copyright for the publications made accessible via the Queen's University Belfast Research Portal is retained by the author(s) and / or other copyright owners and it is a condition of accessing these publications that users recognise and abide by the legal requirements associated with these rights.

Take down policy

The Research Portal is Queen's institutional repository that provides access to Queen's research output. Every effort has been made to ensure that content in the Research Portal does not infringe any person's rights, or applicable UK laws. If you discover content in the Research Portal that you believe breaches copyright or violates any law, please contact openaccess@qub.ac.uk. 


\section{Accepted Manuscript}

On the statistics of transverse permeability of randomly distributed fibers

Masoud Bodaghi, Giuseppe Catalanotti, Nuno Correia

PII:

S0263-8223(16)31319-8

DOI:

http://dx.doi.org/10.1016/j.compstruct.2016.09.045

Reference:

$\operatorname{COST} 7771$

To appear in: $\quad$ Composite Structures

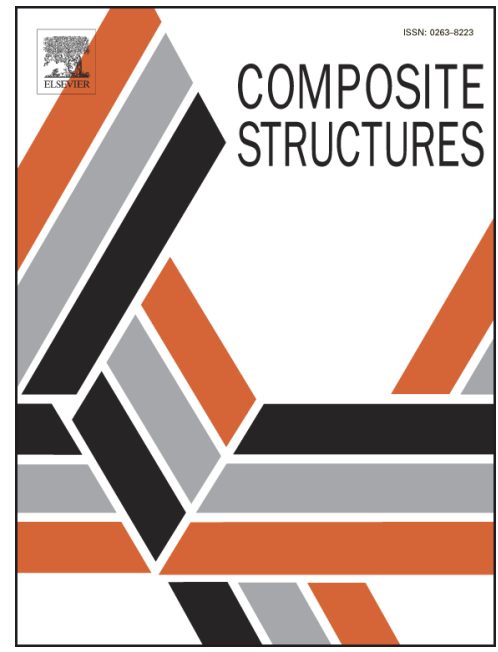

Received Date: $\quad 25$ July 2016

Accepted Date: $\quad 16$ September 2016

Please cite this article as: Bodaghi, M., Catalanotti, G., Correia, N., On the statistics of transverse permeability of randomly distributed fibers, Composite Structures (2016), doi: http://dx.doi.org/10.1016/j.compstruct.2016.09.045

This is a PDF file of an unedited manuscript that has been accepted for publication. As a service to our customers we are providing this early version of the manuscript. The manuscript will undergo copyediting, typesetting, and review of the resulting proof before it is published in its final form. Please note that during the production process errors may be discovered which could affect the content, and all legal disclaimers that apply to the journal pertain. 


\title{
On the statistics of transverse permeability of randomly distributed fibers
}

\author{
Masoud Bodaghi \\ Engineering Design and Advanced Manufacturing, MIT Portugal Program, Faculty of \\ Engineering, University of Porto, Portugal \\ Giuseppe Catalanotti ${ }^{a, b}$, Nuno Correia ${ }^{a, *}$ \\ ${ }^{a}$ INEGI, Institute of Science and Innovation in Mechanical and Industrial Engineering, \\ Portugal \\ ${ }^{b}$ DEMec, Faculdade de Engenharia, Universidade do Porto, Portugal
}

\begin{abstract}
An RVE-based stochastic numerical model is used to calculate the permeability of randomly generated porous media at different values of the fiber volume fraction for the case of transverse flow in a unidirectional ply. Analysis of the numerical results shows that the permeability is not normally distributed. With the aim of proposing a new understanding on this particular topic, permeability data are fitted using both a mixture model and a unimodal distribution. Our findings suggest that permeability can be fitted well using a mixture model based on the lognormal and power law distributions. In case of a unimodal distribution, it is found, using the maximum-likelihood estimation method (MLE), that the generalized extreme value (GEV) distribution represents the best fit. Finally, an expression of the permeability as a function of the fiber volume fraction based on the GEV distribution is discussed in light of the previous results. Keywords: Porosity, Transport properties, Statistics, Finite element analysis (FEA), Tortuosity, Permeability
\end{abstract}

\footnotetext{
* Corresponding author

Email address: ncorreia@inegi.up.pt (Nuno Correia)
} 


\section{Introduction}

The flow of a fluid through porous media is a central problem in many engineering applications such as composite manufacturing, rheology, geophysics, oil engineering, etc. An excellent review of the relevant contributions in the field 5 is reported in [1].

In liquid composite molding (LCM), the porous medium consists of dry fibers, while the fluid is a polymer resin. The knowledge of the transport properties is crucial when setting the manufacturing process parameters, and their determination has been investigated using experimental techniques $[2,3,4,5,6$, 7, 8, 9], numerical simulations such as the lattice Boltzmann method (LBM) [10] or the Finite Element Method (FEM) [11], and analytical solutions [7, 8, 9].

The determination of the transport properties of a fluid through a porous medium is a complicated task and depends on parameters such as pressure gradient, velocity, viscosity and compressibility of the fluid. However, in composites manufacturing several simplifications apply. First of all, the viscosity of the resin is high: in the region of 100-1000 mPa.s for RTM, and the velocity of the fluid is very small, therefore creeping flow (known also as Stokes). Resin can also be considered incompressible so that viscosity, $\mu$, is the only material parameter that plays a role in the determination of the transport properties.

When considering the porous material as homogeneous, and under the hypothesis of creeping flow, Darcy's law applies, i.e. the flow is proportional to the pressure drop and inversely proportional to the fluid viscosity. In the described conditions, fluid motion is described in terms of the permeability, $K$, that depends only on the geometry of the porous medium. On the other hand, at very low Reynolds number (creeping flow), the Carman-Kozeny (CK) equation applies $[12,13]$, and permeability is expressed as a function of the geometry of the particles that form the porous $b^{1}$. If a random distribution of fibers is considered, a modification of CK equation is required [11].

The study of the flow of the resin at a micro-scale level, and the effects of the micro-structure, have already been investigated in previous studies $[2,11]$. 
In those studies the fibers are considered regularly arranged and the effect of random distribution of the fiber is not taken into account.The uncertainties associated with permeability originated from fiber size distribution, porosity and compaction, will cause significant errors [14] when simulating the transport properties of fluid in porous media. Although previous studies have assumed permeability as a random variable in porous media modeling, the complexity involved in establishing a mutual relationship between micro- and macro-scale uncertainties is not common, with only one paper so far [15] proposing a probability density function for permeability.

Nevertheless it is undeniable that the random distribution of the fibers has an effect on the permeability $[16,17,18]$. The aim of this paper is therefore to quantify this effect and point at ways to propose a method to calculate the saturated permeability of the fibrous porous medium.

At the micro-scale, the porous medium consists of several cylindrical fibers,

45 all nearly parallel between them, and randomly distributed. Here, only the transverse permeability is investigated (permeability in the transverse direction of the fiber), the two dimensional case is considered, and two-dimensional Representative Volume Elements (RVEs) are generated to model the fibers as impermeable circles (the capillarity effects and permeability of the fiber is neglected). The RVEs are generated by using the algorithm previously developed by the authors [19] and it is applied to a range of fibre volume fractions. Finally a method for the estimation of the permeability at each fiber volume fraction is proposed.

It should be noted that several micro-structural effects influence permeability, including: i) the shape of the fiber, ii) the size of the fiber and its statistical variation, iii) the volume fraction of the composite and its statistical variation, and iv) the fiber distribution. In this paper we focus on the effects of the random distribution of the fibers alone. 


\section{Methods}

In the case of a stationary creeping flow of a Newtonian incompressible fluid in the absence of body forces, the Stokes equations read:

$$
\begin{aligned}
\nabla \cdot\left[-p \boldsymbol{I}+\mu\left(\nabla \boldsymbol{u}+(\nabla \boldsymbol{u})^{T}\right)\right] & =0 \\
\rho \nabla \cdot \boldsymbol{u} & =0
\end{aligned}
$$

60 where $p$ is the pressure, $\boldsymbol{u}$ is the velocity, $\boldsymbol{I}$ the identity m
density and the dynamic viscosity of the fluid, respectively.

Assuming no voids, superficial velocity $U$ is obtained from the local velocity of the fluid as:

$$
U=\frac{1}{V} \int_{V_{f}} u \mathrm{~d} v=\varepsilon \bar{u}=\left(1-v_{f}\right) \bar{u}
$$

where $V$ is the total volume, $V_{f}$ is the volume of the fluid, $u$ is the local velocity of the fluid, $\bar{u}$ is the average velocity, $\varepsilon$ is the porosity, and $v_{f}$ is the fibre volume fraction. Darcy's law relates the superficial velocity within the RVE to the pressure gradient across the characteristic size of the RVE, $L$ :

$$
U=-\frac{K}{\mu} \nabla p
$$

where $K$ is the permeability of the porous bed and $\nabla p$ is the pressure gradient. Permeability depends only on the geometry of the RVE, and is independent from the superficial velocity and from the pressure. Therefore it can be written:

$$
K=K\left(d, L, v_{f}\right)
$$

where $d$ is the diameter of the fiber, $L$ is the characteristic length of the RVE, and $v_{f}$ the volume fraction. As dimensionally, permeability may be normalised $\tilde{K}=K / d^{2}$. Then equation 4 can be rewritten as:

$$
\tilde{K}=\tilde{K}\left(N_{f}, v_{f}\right)
$$

where $N_{f}=L / r$, being $r$ the radius of the fiber. The normalized permeability in equation (5) is a function of the fiber volume fraction, $v_{f}$, and of the size of the RVE, $N_{f}$. 
The dependence on the size of the RVE can be eliminated if sufficiently big RVEs are used. A preliminary study on the minimum size of the RVE necessary to eliminate $N_{f}$ from equation (5) is conducted, leading to the determination of the normalized permeability as a function of the fibre volume fraction alone, $\tilde{K}=\tilde{K}\left(v_{f}\right)$.

\section{Numerical model}

\subsection{Detail of the FE model}

Computational Fluid Dynamics (CFD) models were created using the commercial software COMSOL Multiphysics 4.4 [20]. Matlab R2013b [21] was used to generate the random distribution of fibers [19] and to run CFD simulations in a FOR loop. 2000 simulations were run at every volume fraction, $v_{f}=0.3,0.35,0.4, . ., 0.8$. A total of 22000 simulations were run to obtain the statistical distribution of the normalized permeability.

RVEs were meshed using triangular mesh elements. In a preliminary phase simulations were run with the aim of choosing the appropriate element dimension. In order to insure convergence, this work verified that the size of the chosen element represented a good compromise between computational cost and accuracy of results. The mesh independence of the results was also verified.

Periodic boundary conditions were applied to the exterior edges of the RVE (in yellow in Figure 1) meanwhile wall boundary conditions were imposed at the interior edges. To impose a periodic boundary condition, the source and sink edges need to be defined. If the flow goes from West (W) to East (E), the boundary conditions are written as (please refer to Figure 1):

$$
\begin{aligned}
& p_{E}-p_{W}=\Delta p \neq 0 \\
& p_{N}-p_{S}=0
\end{aligned}
$$

where $\Delta p$ is the arbitrary pressure drop imposed. Under the assumed hypothesis, in fact, permeability is independent from the applied pressure drop. 


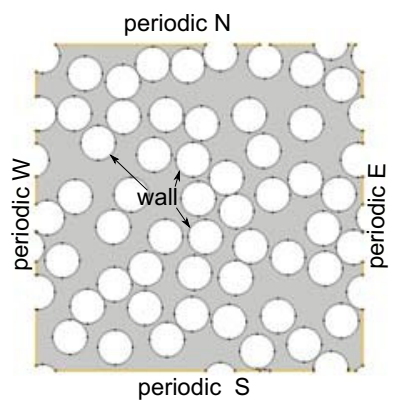

Figure 1: Applied boundary conditions.

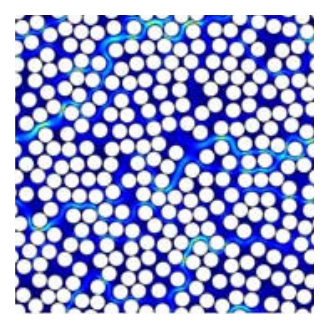

(a) Velocity field.

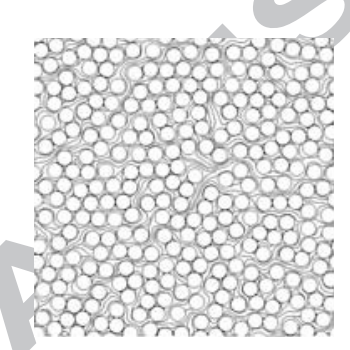

(b) Streamlines.

Figure 2: Results of the numerical model.

After running the simulation, velocity fields and streamlines are obtained as reported in Figures 2(a) and 2(b), respectively. The velocity fields can be integrated (see equation 2) to calculate the superficial velocity $U$. At this point, by knowing the applied pressure drop $\Delta p$, the size of the RVE, and the viscosity of the fluid, the permeability is calculated as shown in equation (3). Streamlines are used to infer the tortuosity, which is defined as the ratio between the length of the streamline and the length of the porous bed. In the present case it is proposed to calculate the tortuosity as:

$$
\tau=\frac{\sum l_{e}^{i}}{\sum l_{p}^{i}}
$$

where $l_{e}^{i}$ is the effective length of the $i$-th streamline, and $l_{p}^{i}$ is the length of the streamline projected along the flow direction. 


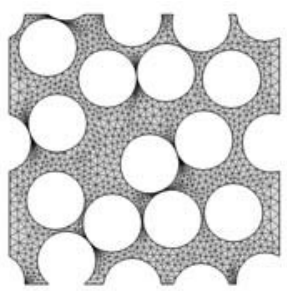

(a) $N_{f}=10$

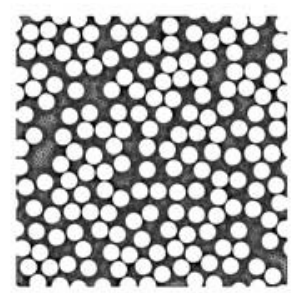

(b) $N_{f}=30$

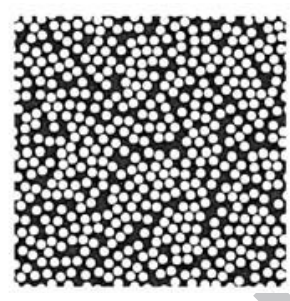

(c) $N_{f}=50$

Figure 3: RVEs' dimensions (not in scale).

\subsection{Size of the RVE}

If the size of the RVE is sufficiently large, or under the assumption of ergodicity, the expected value associated with the permeability distribution, and its standard deviation converge. This has been shown in [16] and it is confirmed by our calculations. Examples of the generated RVEs are reported in Figure 3. Figures 4(a)-4(b) show the probability density function (pdf) of the normalized permeability as a function of the dimension of the RVE. The pdf is calculated using the kernel smoothing estimation based on Gaussian distribution. It is observed that small RVEs may lead to an error in the estimation of the expected value and to big standard deviations. Figures $4(\mathrm{a})-4(\mathrm{~b})$ show that when the size of the RVE is 30 times larger than the radius of the fiber $\left(N_{f} \geq 30\right)$, both the expected value and the standard deviation of the statistical distribution converge. In this study, $N_{f}=40$ is used.

\section{Results and discussion}

\subsection{The normality test}

Matlab Statistical Toolbox [21] and R Statistical Software [22] are used for the statistical analyses performed in the following.

Figure 5 shows the histogram and $\mathrm{Q}-\mathrm{Q}$ plot obtained for $v_{f}=0.5$. It is evident that the normal distribution cannot be an appropriate estimation for the permeability distribution. The same considerations apply to the permeability 


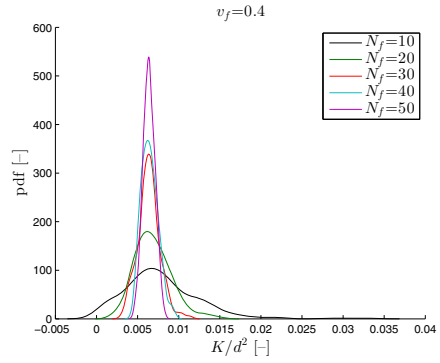

(a) $v_{f}=0.4$

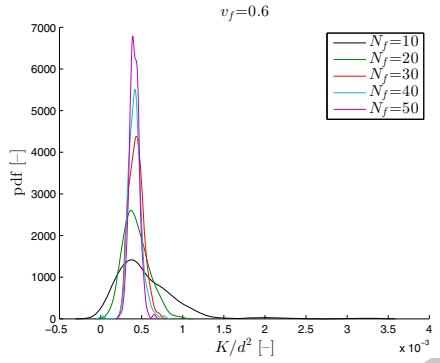

(b) $v_{f}=0.6$

Figure 4: pdfs of the normalized permeability distribution as a function of the size of the RVE.

Table 1: Skewness and kurtosis of the numerical distributions.

\begin{tabular}{lccccccccccc}
$v_{f}$ & 0.3 & 0.35 & 0.4 & 0.45 & 0.5 & 0.55 & 0.6 & 0.65 & 0.7 & 0.75 & 0.8 \\
\hline skewness & 0.21 & 0.21 & $4.8 \mathrm{e}-2$ & 0.19 & 0.23 & 0.21 & 0.16 & 0.20 & 0.31 & 0.38 & 0.15 \\
kurtosis & 3.0 & 2.8 & 2.8 & 2.8 & 3.0 & 2.9 & 2.9 & 2.9 & 2.9 & 2.5 & 2.9
\end{tabular}

distributions obtained for different values of the volume fraction. However, for the sake of conciseness, Q-Q plots concerning these distributions are not reported here. From Figure 5 it can be observed that permeability distributions show fatter tails and thinner body than the normal distribution (Figure 5(a)), and that it is approximately symmetric (Figure 5(b)). It should be noticed that the true and normal density (Figure 5(b)) cross each other twice on each side of the mean value, satisfying Finuncan's condition [23] which states that fatter tails are often associated to more peaked distributions. Moreover, with reference to [24], the difference in the appearance of the distributions is due to the difference in variances and not in kurtosis. This is shown in Table 1, where skewness and kurtosis of the permeability data are reported. Table 1 shows that the skewnesses of the permeability data lies between 0 and 0.5 , implying that the permeability distribution is approximately symmetric. In addition, the kurtosis values are around 3 which is consistent with a normal distribution. As expected, there is no significant difference between the calculated kurtosis and that of the normal distribution and hence this method does not allow one to 


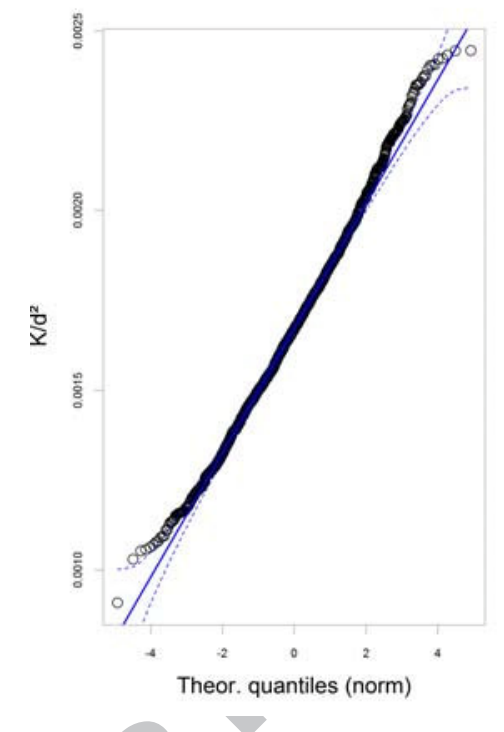

(a) Normal distribution fit (dashed lines indicate the $95 \%$ confidence interval using the KS test)
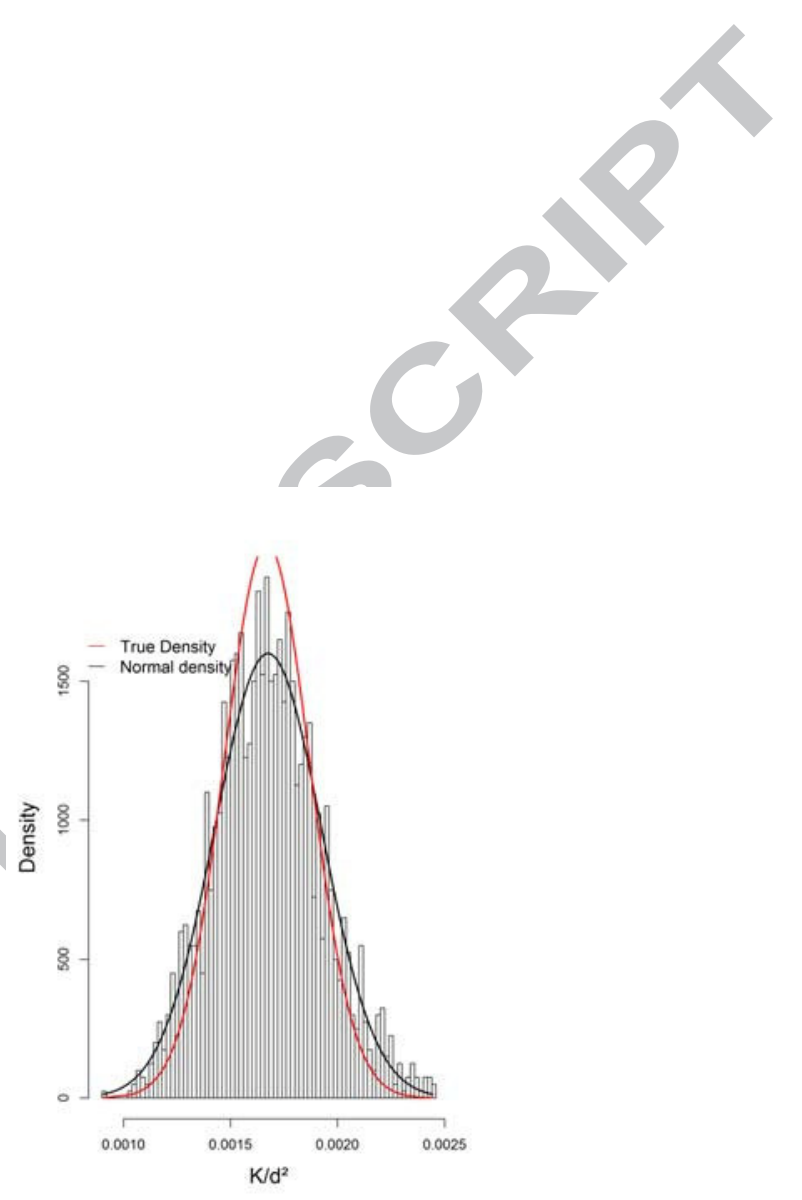

(b) Histogram, normal fit (in black) and true density (in red)

Figure 5: Q-Q plots and pdf of permeability data $\left(v_{f}=0.5\right)$. 
Table 2: Statistical characterizations of permeability data derived from RVEs

\begin{tabular}{ccccccc}
\hline$v_{f}$ & $\tau($ mean $)$ & $C O V(\tau)$ & $\tilde{K}$ (mean) & COV $(\tilde{K})$ & Chi-square test significance level \\
\hline & & & & & Normal & Lognormal \\
0.3 & 1.34 & 0.0074 & 0.024 & 0.131 & 0.0779 & 0.0029 \\
0.4 & 1.42 & 0.007 & 0.0064 & 0.145 & 0.00013 & $1.89 \mathrm{E}-10$ \\
0.5 & 1.47 & 0.108 & 0.0016 & 0.148 & 0.042 & 0.027 \\
0.6 & 1.51 & 0.08 & 0.00047 & 0.157 & 0.2438 & 0.003262 \\
0.7 & 1.54 & 0.008 & 0.00012 & 0.161 & 0.3 & $2.10 \mathrm{E}-09$ \\
0.8 & 1.53 & 0.006 & $1.83 \mathrm{E}-05$ & 0.125 & 0.1558 & 0.05813 \\
\hline
\end{tabular}

detect the large variation in the tails [23].

\subsection{Permeability statistics}

Statistical characterizations of the derived permeability for different values of $v_{f}$ are summarized in Table 2 . Table 2 shows that permeability coefficients of variation $(\mathrm{COVs})$ for different values of $v_{f}$ lie between 0.12 and 0.16 , which is approximately 10 times larger than the tortuosity COVs. These observations suggest that uncertainty associated with permeability is one order of magnitude greater than that associated with tortuosity. Thus, although tortuosity influences the mean value of permeability, it does not explain the uncertainty of permeability. It is thought that, in the present case, the larger uncertainty of permeability is caused by the porosity distribution. In addition, the coefficient of variation for permeabilities is approximately in the same range as reported in $[25,26]$ for smaller sample sizes.

The chi-square test, with a significance of $5 \%$, was also applied to permeability data in terms of normal and lognormal distributions ${ }^{2}$. The $p$ values reported in Table 2 suggest the appropriateness of using a lognormal distribution, over a normal distribution, to represent permeability data. However, a close observation of Figure 6 allows one to conclude that although the true density and lognormal fit are virtually the same (as shown in Figure 6(b)), the lognormal 


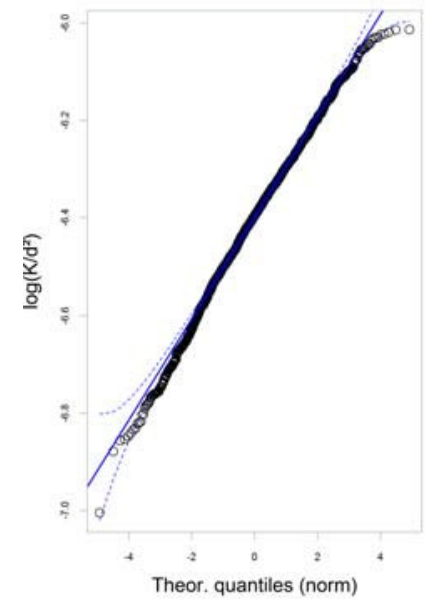

(a) Lognormal distribution fit (dashed lines indicate the $95 \%$ confidence interval using the KS test)

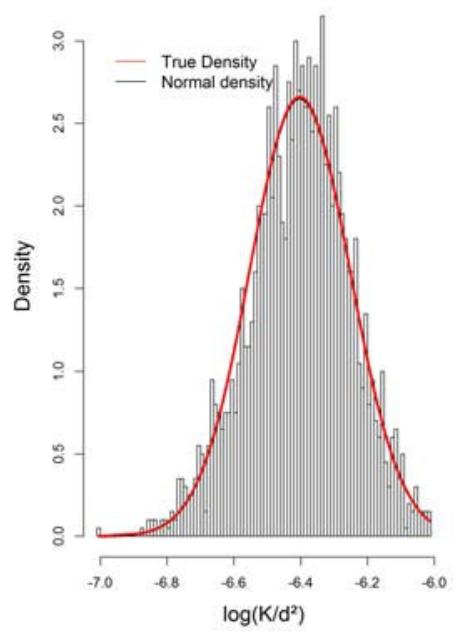

(b) Histogram, normal fit (in black) and true density (in red)

Figure 6: Q-Q plots and pdf of permeability data after log transformation $\left(v_{f}=0.5\right)$.

distribution cannot represent the whole set of data. In fact, even after logarithmic transformation, the distribution shows a substantial skewness, as reported in Figure 6(a). Moreover, from the Q-Q plot of Figure 6(a), it can be concluded that both upper and lower tails fail to represent the permeability at very low probability.

Summarizing, the lognormal distribution can be used to fit the data of the body of the empirical distribution, but fails at low probability (i.e. in the tails). To take into account the tails, extreme value theory is used. In the following, two approaches are proposed to fit the permeability data: i) the use of a mixture of two or more distributions [27], ii) the use of heavy-tailed distribution models. 


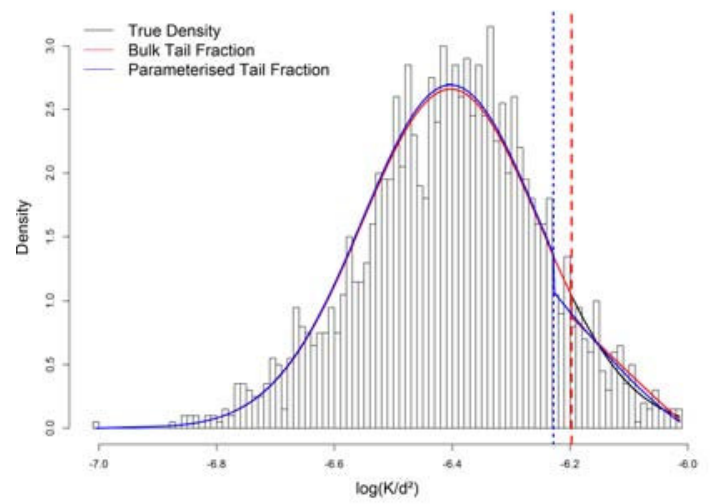

Figure 7: Histogram of permeability data at $v_{f}=0.5$ with parametric estimation of tail: the bulk tail fraction in red fitted with normal and parameterised tail fraction in blue fitted with Generalized Pareto distribution. The vertical dashed lines indicated the threshold.

\subsubsection{Mixture modelling and threshold estimation}

This paper proposes to define two different distributions to fit the body and the tails of the empirical distribution. In the previous section it was concluded that the lognormal distribution fits well the empirical data in the body of the distribution; therefore, for the body the lognormal distribution is used. For the tails, a power distribution is used. The reason is that, in the framework of extreme values theory [28], it has already been shown that power distribution is the best choice for fat tails.

Figure 7 shows the mixture model of the permeability data for $v_{f}=0.5$. The estimation of the threshold (represented as vertical dashed lines in Figure 7) where the transition from one model to another occurs, is critical. This transition exists in both the left and right tails of the empirical distribution, but in Figure 7 only the upper threshold is represented for clarity. Mathematically, a probability distribution of the permeability data following a power law model can be expressed as equation (8):

$$
P_{p}(K)=\alpha \tilde{K}_{\min }^{\alpha} K^{-(\alpha+1)}
$$

where $\alpha$ and $\tilde{K}_{\min }$ are experimental positive constants. For the lognormal 
distribution it reads:

$$
P_{l n}(K)=\frac{1}{\sigma \sqrt{2 \pi}} \frac{1}{\tilde{K}} \exp \frac{-(\ln \tilde{K}-\mu)^{2}}{2 \sigma^{2}}
$$

Taking the logarithm of (8) and (9) yields:

$$
\begin{aligned}
& \ln \left(P_{p}(K)\right)=\ln (\alpha)+\alpha \ln \left(\tilde{K}_{\text {min }}\right)-(\alpha+1) \ln (K) \\
& \ln \left(P_{l n}(K)\right)=\ln (\sigma \sqrt{2 \pi})-\ln (K)-\frac{(\ln (K)-\mu)^{2}}{2 \sigma^{2}}
\end{aligned}
$$
conclude that an appropriate permeability distribution model closely fits the body of a lognormal distribution and the tails of the double Pareto distribution.

It is not clear what could cause the fat tails. Strictly speaking, fiber distribution is not completely random. It is clear that Complete Spatial Randomness 180

In case of large $\sigma$, equation (11) would show near-linear log-density beahviour. Therefore both the power and lognormal distributions would have near-linear $\log$ Complementary Cumulative Distribution Functions, lccdf.

The lccdf of the transformed data is plotted for $v_{f}=0.5$ in Figure 8.

As expected, the body of the empirical distribution can be fitted using a lognormal model while the tails are fitted using a power law model in [29] (blue and red lines in Figure 8, respectively). Therefore the permeability data can be fitted using a linear combination of the lognormal and power law models: in the range $\tilde{K}_{\min } \leq \tilde{K} \leq \tilde{K}_{\max }$ the permeability data can be fitted using a lognormal model, while outside this range $\left(\tilde{K}<\tilde{K}_{\min }\right.$ and $\left.\tilde{K}>\tilde{K}_{\max }\right)$ the permeability data is fitted using a power law model. This behaviour was already observed by [30] who showed that a mixture of lognormal distributions in terms of a geometric distribution would behave lognormally in the body and have a power law model in the tails. More recently, the above model was corrected by a double Pareto distribution [31] who showed that the mixture distribution specifically have Paretian (power law) behavior in the tails. Hence, one could (CSR) cannot be achieved, as found already in [19]: due to the finite size of fibers, their distribution cannot be completely random because the space occupied is limited by others (it should be noticed that for the generation of the 


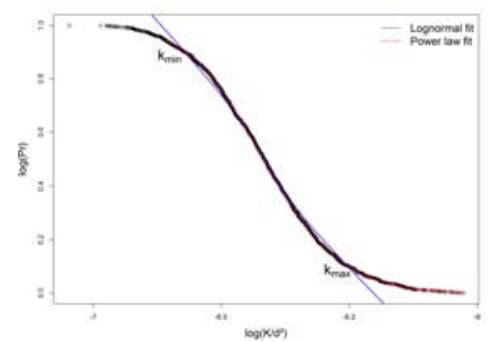

Figure 8: Distribution of the transformed permeability data at $\mathrm{vf}=0.5$ (double logarithmic scale).

RVE a hard-core model is used, therefore interpenetration between fibers cannot occur).

\subsubsection{Fitting extreme value distributions}

Statistical tests allow one to reject the null hypothesis that a sample comes from a given statistical distribution but do not determine from which distribution a given sample comes from. Of course this is not a simple task and even if several statistical methods (as the methods of moments) can be applied to this purpose, the determination of the distribution cannot be rigorous.

Nonetheless, this paper proposes to obtain the candidate statistical distribution as the distribution that best fit the numerical data. Several distributions were used to fit the data.In particular: beta, birnbaumsaunders, exponential, extreme value, gamma, generalized extreme value, generalized pareto, inverse

gaussian, logistic, loglogistic, nakagami, normal, rayleigh, rician, tlocationscale, and weibull were studied.

The best fit was obtained using the maximum-likelihood estimation (MLE) method and minimizing the negative logarithm of the likelihood.

Figure 9 shows the empirical pdf and the best fits (among the distributions 200 aforementioned). The distributions are listed in the legend in order of best fit.

It is possible to observe that the generalized extreme value (GEV) represents the best fit the data for all the volume fractions considered. 


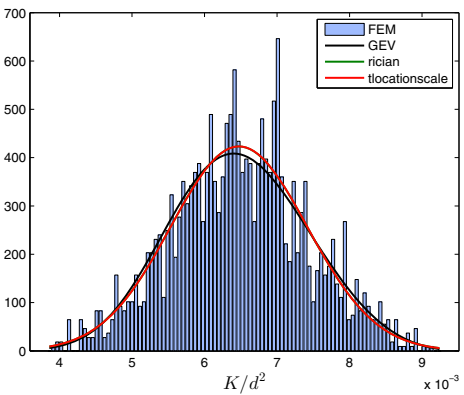

(a) $v_{f}=0.4$

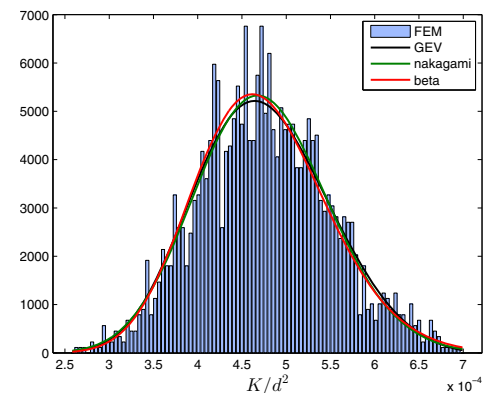

(b) $v_{f}=0.6$

Figure 9: pdfs of the normalized permeability distributions (for the sake of conciseness the distributions are reported only for $\left.v_{f}=0.4,0.6\right)$.

\section{Correlation between tortuosity, porosity and permeability}

In the previous sections, it was shown that a mixture model (consisting of a

lognormal distribution for the body and a power law model (or pareto [29]) for the tails) or a GEV provide better fit over normal distributions for permeability of macroscopic samples with randomly arranged fibers.

These families of distributions (GEV, power law, pareto, lognormal) have been found to be useful for stochastic modelling $[32,33,34,35,36]$ when inputs such as permeability follow heavy-tailed distributions. However, the use of mixture model is more complex than GEV. Thus, for the sake of convenience, the GEV model is used in the following because it i) provides a closed-form expression, ii) yields a permeability model with two parameters, iii) encompasses a range of classes of tail behaviour.

The pdf of the GEV distribution reads:

$$
f=\frac{1}{\sigma}\left[1+\kappa\left(\frac{x-\mu}{\sigma}\right)\right]^{-1-\frac{1}{\kappa}} \exp \left\{-\left[1+\kappa\left(\frac{x-\mu}{\sigma}\right)\right]^{-\frac{1}{\kappa}}\right\}
$$

where $\mu, \sigma$, and $\kappa$ are the location parameter, the scale parameter, and the shape parameter for the GEV distribution, respectively. Figure 10 shows the variation of $\mu$ and $\sigma$ as a function of the volume fraction. The location, the 


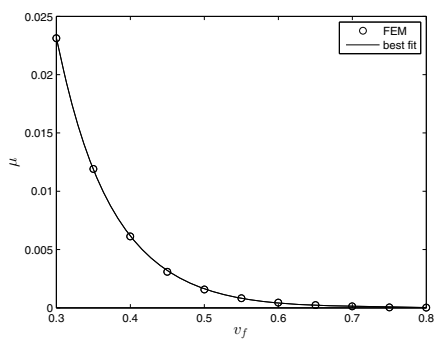

(a) $\mu$

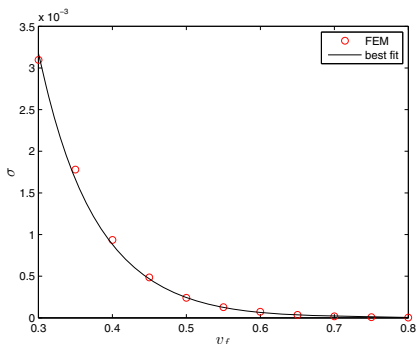

(b) $\sigma$

Figure 10: Location and scale parameters as a function of the volume fraction.

scale, and the shape parameter can be fitted as:

$$
\begin{aligned}
& \mu=1.232 \exp \left(-13.25 v_{f}\right) \\
& \sigma=0.1496 \exp \left(-12.84 v_{f}\right) \\
& \kappa \approx-0.215
\end{aligned}
$$

as the shape parameter is approximately constant when varying the fiber volume fraction. Equations (13) allow the calculation of permeability as a function of the fiber volume fraction. The normalized permeability is obtained as the expected value of the GEV distribution and it reads:

$$
\frac{K}{d^{2}}=E\left(x ; v_{f}\right)=\mu-\frac{\sigma}{\kappa}+\frac{\sigma}{\kappa} \Gamma(1-\kappa)
$$

where $\Gamma(\bullet)$ is the gamma function of $(\bullet)$. Figure 11 shows the normalized permeability as a function of the volume fraction and the confidence interval at 95\%. Permeability can also be obtained by the Carman-Kozeny equation [12] as:

$$
K=\frac{\varepsilon \mu u}{\nabla p}=\frac{\varepsilon D_{h}^{2}}{\psi_{C K}}
$$

where $D_{h}=4 / d[11]$ is the hydraulic diameter and $\psi_{C K}$ is the Kozeny factor. Substituting the expression of the hydraulic diameter in equation (15) and rearranging yields:

$$
\psi_{C K}=\frac{d^{2}\left(1-v_{f}\right)^{3}}{K v_{f}^{2}}=\frac{d^{2} \varepsilon^{3}}{K(1-\varepsilon)^{2}}
$$




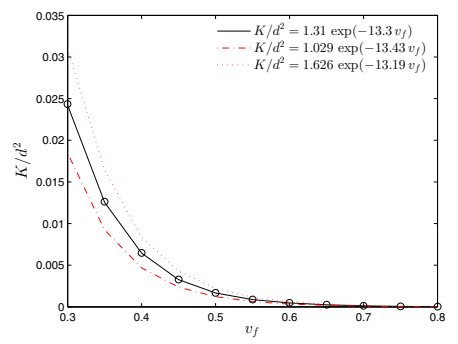

Figure 11: Normalized permeability as a function of the volume fraction.

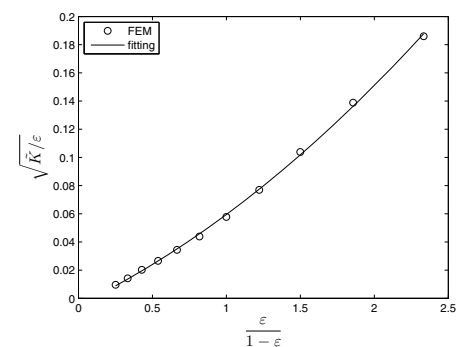

(a) $\sqrt{\tilde{K} / \varepsilon}$ vs. $\varepsilon /(1-\varepsilon)$.

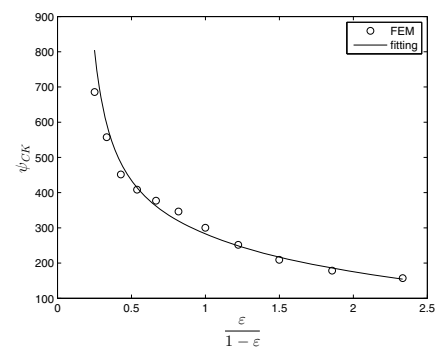

(b) $\psi_{C K}$ vs. $\varepsilon /(1-\varepsilon)$.

Figure 12: Determination of the Kozeny factor.

The Kozeny factor is a dimensionless parameter usually obtained experimentally. Substituting equation (14) in (16) allows the calculation of the Kozeny factor as a function of $\varepsilon /(1-\varepsilon)$ as reported in Figure 12. The Kozeny factor can be fitted as:

$$
\psi_{C K}=\left(0.01374 \frac{\varepsilon}{1-\varepsilon}+0.05038-0.004641 \frac{1-\varepsilon}{\varepsilon}\right)^{-2}
$$

At this point, it is worth comparing the Kozeny factor, $\psi_{C K}$, calculated using equation (17), with both experiments and models found in the literature. Figure 13 shows this comparison considering models and experiments reported by other authors $[2,11,16,37,38,39,40,41,42]$. It is noted that the proposed model correlates well with the original CK and Gebart's model [2] only for large values of porosity. On the contrary, for small values of the porosity, the results of our model do not match the prevision of Gebart's model [2] but they correlate better with the experiments. As proposed by Carman [12] the Kozeny factor 

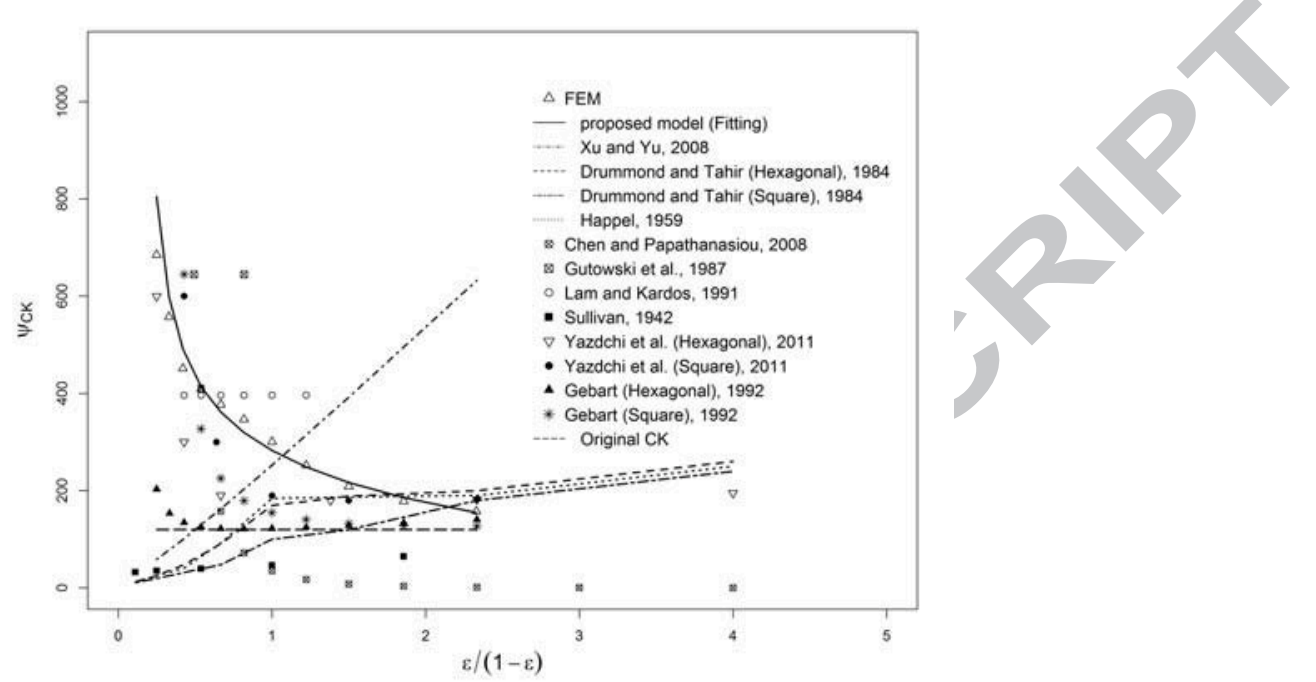

Figure 13: Comparison of the proposed model with experiments and models found in literature.

can also be written as:

$$
\psi_{C K}=\Phi \tau^{2}
$$

where $\tau$ is the tortuosity and $\Phi$ is a parameter taking into account the shape of the particles (or the fibers). The tortuosity was estimated by Carman [12] to be constant and equal to $\sqrt{2} \approx 1.4$ for a porous bed composed by spherical particles. The tortuosity calculated using equation (7) is reported in figure 14 as a function of $v_{f}$. Please, notice that the value proposed by Carman does not substantially differs from the value calculated here for the case of cylindrical fibers. The tortuosity calculated can be fitted as:

$$
\tau=1.552 \exp \left\{-\left[\left(v_{f}-0.7787\right) / 1.221\right]^{2}\right\}
$$

Finally the shape parameter $\Phi$ is calculated from equation (18). Figure 15 shows the variation of $\Phi$ with the volume fraction $v_{f}$. It can be fitted as:

$$
\Phi=81.33\left(\frac{\varepsilon}{1-\varepsilon}\right)^{-0.7508}+48.67
$$




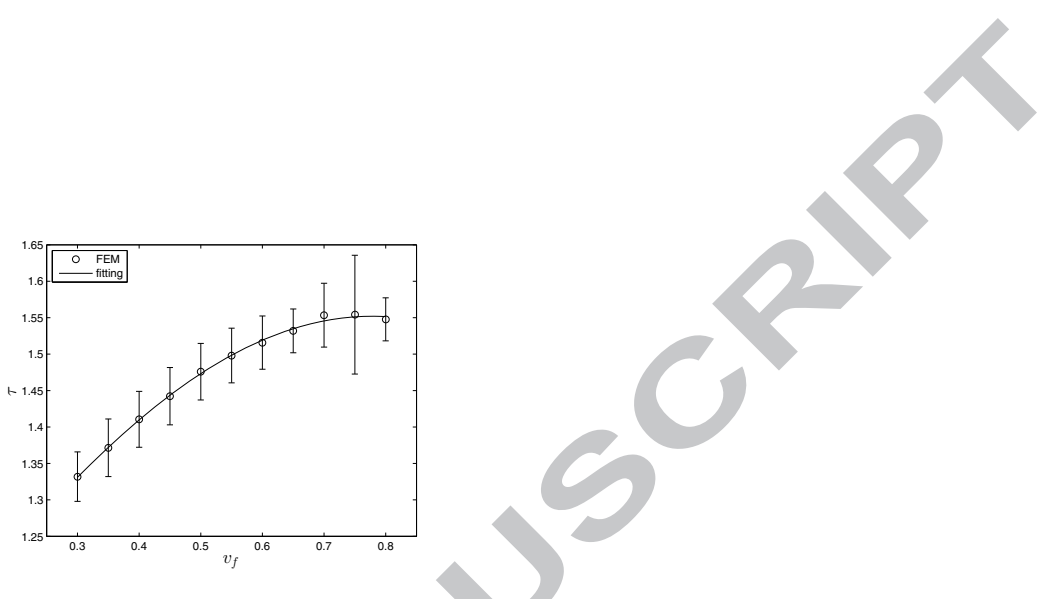

Figure 14: Tortuosity as a function of the volume fraction. Error bars represent the standard deviation.

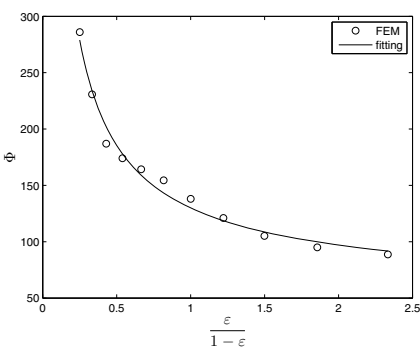

Figure 15: $\Phi$ as a function of $v_{f}$. 


\section{Conclusion}

The main conclusions of this work may be summarized in the following points.

- It was demonstrated that using CFD simulations and applying appropriate boundary conditions is possible to obtain the statistical distribution of the saturated permeability of a fibrous porous medium. Only the saturated transverse permeability was considered in this paper. The uncertainty in the calculation of permeability depends on several parameters: 1) the uncertainty in the measure of the volume fraction, 2) the uncertainty in the dimension of the fibers (fiber diameter is not constant but varies); 3) the effect of the random distribution of the fiber. Only the last effect was considered here.

- The normalized permeability distribution appears not to be normal. Q-Q plots showed that the permeability distribution has fatter tails than the normal distribution. The fact that the normalized permeability is not normally distributed may be surprising but it may be explained with the fact that the position occupied by the fibers is not completely random. Complete Spatial Randomness (CSR) exists only for points generated in a region using a Poisson distribution. Circles, having a finite dimensions do not allow CSR because of the fact that the space occupied by a given circle is unavailable to other circles [19].

- It is common practice to ignore the extreme values, as done in the second worldwide permeability benchmark [25]. Our results, however, showed that extreme values in permeability data cannot be considered outliers with negligible probability and that a normal distribution cannot be the characteristic model for permeability.

- We also showed how to identify both the lower and upper tails in the data. We postulate that the reasons for this non-normal behaviour could be, i) a combination of micro- and macro-scale flows, ii) undetectable patterns of 
permeability because of heterogeneous distributions of fibers, iii) random distribution of fibers.

- We propose that a mixture model (consisting of a lognormal distribution for the body and a power law model (or pareto [29]) for the tails) or a GEV provide better fit for the description of the statistics of permeability.

- An expression of the permeability and of other relevant parameters used in Carman-Kozeny equation, was obtained as a function of the volume fraction $v_{f}$.

\section{Acknowledgement}

The authors would like to acknowledge support from the Portuguese Foundation for Science and Technology, under the research Grant SFRH/BD/51578/2012 and the MIT-Portugal Program. The authors would like to thank project SciTech, reference NORTE-01-0145-FEDER-000022, within the Portugal 2020 (PT2020) in the NORTE-45-2015-02 program, through the European Regional Development Fund (ERDF).

\section{Notes}

${ }^{1}$ Strictly speaking, Carman-Kozeny (CK) equation applies to porous beds of spherical particles, but it has been applied also to the case of fibrous media.

${ }^{2}$ Chi-square test rejects the null-hypothesis when the $p$ value is $p>0.05$.

\section{References}

[1] T. a. K. Sadiq, S. G. Advani, R. S. Parnas, Experimental Investigation of Transverse Flow-through Aligned Cylinders, International Journal of Multiphase Flow 21 (5) (1995) 755-774. doi :Doi10.1016/0301-9322(95)00026-T. 
[2] B. Gebart, Permeability of Unidirectional Reinforcements for RTM, Journal of Composite Materials 26 (8) (1992) 1100-1133. arXiv:http://jcm.sagepub.com/content/26/8/1100.full.pdf+html, doi:10.1177/002199839202600802.

[3] R. S. Parnas, A. J. Salem, A comparison of the unidirectional and radial in-plane flow of fluids through woven composite reinforcements, Polymer Composites 14 (5) (1993) 383-394. doi:10.1002/pc.750140504.

[4] C.-H. Wu, T. James Wang, L. James Lee, Trans-plane fluid permeability measurement and its applications in liquid composite molding, Polymer Composites 15 (4) (1994) 289-298. doi:10.1002/pc.750150407.

[5] B. R. Gebart, P. Lidström, Measurement of in-plane permeability of anisotropic fiber reinforcements, Polymer Composites 17 (1) (1996) 43-51. doi:10.1002/pc.10589.

[6] C.-H. Shih, L. J. Lee, Effect of fiber architecture on permeability in liquid composite molding, Polymer Composites 19 (5) (1998) 626-639. doi:10.1002/pc.10136.

[7] B. Yu, L. James Lee, A simplified in-plane permeability model for textile fabrics, Polymer Composites 21 (5) (2000) 660-685. doi:10.1002/pc.10221.

[8] B. Yu, H.-T. Chiu, Z. Ding, L. James Lee, Analysis of heat tranfer and flow in liquid composite molding, Polym. Process. 15 (53) (2002) 273-283. doi:10.3139/217.1592.

[9] B. Yu, L. J. Lee, H. Cao, A fractal in-plane permeability model for fabrics, Polymer Composites 23 (2) (2002) 201-221. doi:10.1002/pc.10426.

[10] E. Belov, S. Lomov, I. Verpoest, T. Peters, D. Roose, R. Parnas, K. Hoes, H. Sol, Modelling of permeability of textile reinforcements: lattice boltzmann method, Composites Science and Technology 64 (7-8) (2004) 10691080. doi:http://dx.doi.org/10.1016/j.compscitech.2003.09.015. 
[11] K. Yazdchi, S. Srivastava, S. Luding, Microstructural effects on the permeability of periodic fibrous porous media, International Journal of Multiphase Flow 37 (8) (2011) 956-966. doi:http://dx.doi.org/10.1016/j.ijmultiphaseflow.2011.05.003.

[12] P. Carman, Fluid flow through granular beds, Trans. Instn Chem. Engrs 15 (1937) 32-48.

[13] P. Carman, Flow of gases through porous media, Butterworths, London, 1956.

[14] M. Bodaghi, C. Gonçalves, N. Correia, A quantitative evaluation of the uncertainty of permeability measurements in constant thickness bre reinforcement, in: Proceedings of ECCM-16 Conference, 2014.

[15] C. B. F. Zhang, S. Comas-Cardona, Statistical modelling of inplane permeability of non-woven random fibrous reinforcement, Composites Science and Technology 72 (12) (2012) 1368-1379. doi:10.1016/j. compscitech.2012.05.008.

[16] X. Chen, T. D. Papathanasiou, The transverse permeability of disordered fiber arrays: a statistical correlation in terms of the mean nearest interfiber spacing, Transport in Porous Media 71 (2) (2008) 233-251. doi:10.1007/s11242-007-9123-6.

[17] Y. Matsumura, T. L. Jackson, Numerical simulation of fluid flow through random packs of polydisperse cylinders, Physics of Fluids 26 (12) (2014)-. doi:http://dx.doi.org/10.1063/1.4903954.

[18] Y. Matsumura, T. L. Jackson, Numerical simulation of fluid flow through random packs of cylinders using immersed boundary method, Physics of Fluids 26 (4) (2014) -. doi:http://dx.doi.org/10.1063/1.4870246.

[19] G. Catalanotti, On the generation of rve-based models of composites reinforced with long fibres or spherical particles., Composite StructuresIn press. doi:10.1016/j. compstruct.2015.11.039. 
[20] COMSOL, COMSOL Documentation, COMSOL Inc. (2015).

[21] MATLAB, MATLAB R2013b, The MathWorks, Inc., Natick, Massachusetts, United States. (2013).

[22] R Core Team, R: A Language and Environment for Statistical Computing, R Foundation for Statistical Computing, Vienna, Austria (2013).

[23] K. P. Balandaa, H. L. Macgillivray, Kurtosis: A critical review, The American Statistician 42 (2) (1987) 111-119. doi : 10.1080/00031305.1988.10475539.

[24] H. L. DeCarlo, On the meaning and use of kurtosis, Psychological Methods 2 (3) (1997) 292-307. doi:10.1037/1082-989X.2.3.292.

[25] R. Arbter, J. Beraud, C. Binetruy, L. Bizet, J. Bréard, S. Comas-Cardona, C. Demaria, A. Endruweit, P. Ermanni, F. Gommer, S. Hasanovic, P. Henrat, F. Klunker, B. Laine, S. Lavanchy, S. Lomov, A. Long, V. Michaud, G. Morren, E. Ruiz, H. Sol, F. Trochu, B. Verl${ }_{340}$ eye, M. Wietgrefe, W. Wu, G. Ziegmann, Experimental determination of the permeability of textiles: A benchmark exercise, Composites Part A: Applied Science and Manufacturing 42 (9) (2011) 1157-1168. doi : http://dx.doi.org/10.1016/j. compositesa.2011.04.021.

[26] N. Vernet, E. Ruiz, S. Advani, J. Alms, M. Aubert, M. Barburski, B. Barari, J. Beraud, D. Berg, N. Correia, M. Danzi, T. Delaviï£ · re, M. Dickert, C. D. Fratta, A. Endruweit, P. Ermanni, G. Francucci, J. Garcia, A. George, C. Hahn, F. Klunker, S. Lomov, A. Long, B. Louis, J. Maldonado, R. Meier, V. Michaud, H. Perrin, K. Pillai, E. Rodriguez, F. Trochu, S. Verheyden, M. Wietgrefe, W. Xiong, S. Zaremba, G. Ziegmann, Experimental determination of the permeability of engineering textiles: Benchmark ii, Composites Part A: Applied Science and Manufacturing 61 (0) (2014) 172-184. doi:http://dx.doi.org/10.1016/j. compositesa. 2014.02.010. 
[27] W. Reed, M.Jorgensen, The double pareto-lognormal distribution

[33] F. Zhang, B. Cosson, S. Comas-Cardona, C. Binetruy, Efficient stochastic simulation approach for $\{\mathrm{RTM}\}$ process with random fibrous permeability, Composites Science and Technology 71 (12) (2011) 1478-1485. doi:http://dx.doi.org/10.1016/j.compscitech.2011.06.006.

[34] F. Zhang, S. Comas-Cardona, C. Binetruy, Statistical modeling of in-plane permeability of non-woven random fibrous reinforcement, doi:http://dx.doi.org/10.1016/j.compscitech.2012.05.008. 
[35] S. Padmanabhan, R. Pitchumani, Stochastic modeling of nonisothermal flow during resin transfer molding, International Journal of Heat and Mass Transfer 42 (16) (1999) 3057-3070. doi:http://dx.doi.org/10.1016/S0017-9310(98)00377-9.

[36] T. Mesogitis, A. Skordos, A. Long, Uncertainty in the manufacturing of fibrous thermosetting composites: A review, Composites Part A: Applied Science and Manufacturing 57 (0) (2014) 67-75. doi:http://dx.doi.org/10.1016/j.compositesa.2013.11.004.

[37] R. R. Sullivan, Specific surface measurements on compact bundles of parallel fibers, Journal of Applied Physics 13 (11) (1942) 725-730. doi:http://dx.doi.org/10.1063/1.1714824.

[38] J. Happel, Viscous flow relative to arrays of cylinders, AIChE Journal 5 (2) (1959) 174-177. doi:10.1002/aic.690050211.

[39] J. Drummond, M. Tahir, Laminar viscous flow through regular arrays of parallel solid cylinders, International Journal of Multiphase Flow $10 \quad$ (5) (1984) $515 \quad$ - 540. doi:http://dx.doi.org/10.1016/0301-9322(84)90079-X.

[40] T. Gutowski, Z. Cai, S. Bauer, D. Boucher, J. Kingery, S. Wineman, Consolidation experiments for laminate composites, Journal of Composite Materials 21 (7) (1987) 650-669. arXiv:http://jcm.sagepub.com/content/21/7/650.full.pdf+html, doi:10.1177/002199838702100705.

[41] R. C. Lam, J. L. Kardos, The permeability and compressibility of aligned and cross-plied carbon fiber beds during processing of composites, Polymer Engineering \& Science 31 (14) (1991) 1064-1070. doi:10.1002/pen.760311411.

[42] P. Xu, B. Yu, Developing a new form of permeability and kozenyï£ · carman constant for homogeneous porous media by means of fractal ge- 
ometry, Advances in Water Resources 31 (1) (2008) 74 - 81. doi:http://dx.doi.org/10.1016/j.advwatres.2007.06.003.

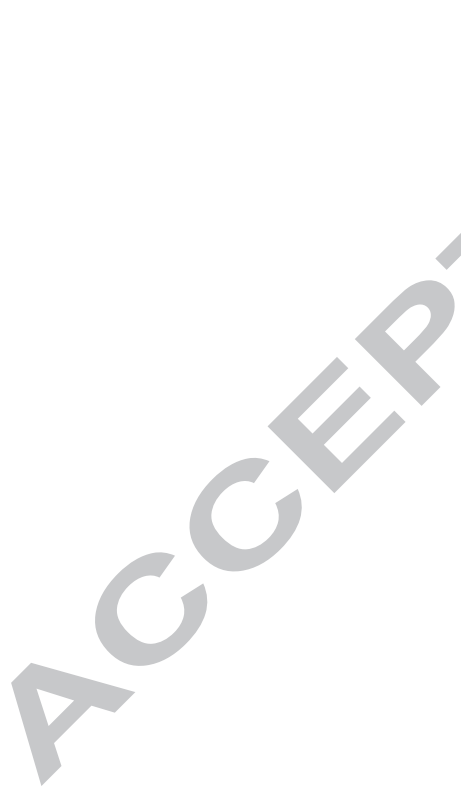

